\title{
BMJ Open Obesity among children and adolescents in Qatar: protocol for a scoping review
}

To cite: Alisi M. Obesity among children and adolescents in Qatar: protocol for a scoping review. BMJ Open 2022;12:e057427. doi:10.1136/ bmjopen-2021-057427

- Prepublication history and additional supplemental material for this paper are available online. To view these files, please visit the journal online (http://dx.doi.org/10.1136/ bmjopen-2021-057427)

Received 16 September 2021 Accepted 02 February 2022

Check for updates

(C) Author(s) (or their employer(s)) 2022. Re-use permitted under CC BY-NC. No commercial re-use. See rights and permissions. Published by BMJ.

Department of Social \& Policy Sciences, University of Bath Department of Social \& Policy Sciences, Bath, UK

Correspondence to

Mariam Alisi;

ma2290@bath.ac.uk

\section{ABSTRACT}

Introduction Approximately half of the school-aged children are overweight or obese in Qatar. Childhood obesity is associated with health conditions in adulthood, such as hypertension, diabetes, metabolic defects, cardiovascular risks and psychological challenges. Existing obesity studies have limitations; thus, there is a need for further research with improved quality, wider representation of population including children and adolescents and methodological diversity. As Qatar moves towards a preventive evidence-based approach in tackling obesity, this scoping review aims to support the efforts of obesity prevention among children and adolescents in Qatar by providing an up-to-date research map that systematically highlights existing knowledge, research gaps and methodological range. In addition, this will be the first scoping review that analyses how obesity research has evolved in Qatar since 2000.

Methods and analysis This scoping review follows the guidelines of Joanna Briggs Institute (JBI) and Preferred Reporting Items for Systematic Reviews and Meta-Analyses Extension for Scoping Reviews for both developing the protocol and reporting the findings. It will use the System for the Unified Management, Assessment and Review of Information-JBI software for synthesis. The review will include any study in Arabic or English published between 1 January 2000 and 31 December 2021 on obesity/overweight among children and/or adolescents in Qatar. Studies without specific data on the concept of obesity, the population of children and adolescents or the context of Qatar will be excluded. The following databases will be searched: PubMed, Embase, Web of Science, IBSS, Scopus, J-STOR, APA PsycNET and Google Scholar. In addition, Arabic literature via Dar Almanthomah, Dar Almanhal and E-Marefah will be searched. A search strategy to identify grey literature will be adopted for a wider reach. The screening process will be conducted by two independent reviewers. Extracted data will be analysed descriptively and analytically using simple frequency counts and qualitative content analysis. The results will be presented using maps, tables and diagrams. Ethics and dissemination Ethics approval is not required as data will be collected from publicly available published research. The results of this review will be disseminated via a peer-reviewed publication, national and international conferences, workshops and knowledge mobilisation activities among key stakeholders.

\section{INTRODUCTION}

The prevalence of obesity in Arab Gulf countries places these countries among those
Strengths and limitations of this study

- This review will be the first scoping review to identify the gaps in this broad topic and the first review to analyse the longitudinal development in research on obesity among children and adolescents in Qatar.

- Employing Joanna Briggs Institute 2020 guidelines for scoping reviews will ensure methodological robustness and transparency.

- The number of existing studies on this topic indicates that the topic is under-researched, and as such, indepth analyses of existing studies are required.

- Some relevant data may not be included in this review as studies on diabetes and cancer indirectly focused on obesity will be excluded.

- Due to the exploratory nature of scoping reviews, the studies that will be included in this review will not be inspected for quality or risk of bias.

with the highest number of individuals with obesity globally. In Qatar, for instance, almost half of the school-aged children are overweight or obese $(46 \%) .^{2}$ Most clinical studies have shown that childhood obesity is linked to adverse effects in adulthood, including hypertension, diabetes, metabolic defects, cardiovascular risks and psychological challenges. ${ }^{3}$ Therefore, childhood obesity is a public health issue, and the population's overall health status can be assessed using its obesity rates. ${ }^{1}$

Although obesity can be a genetic abnormality, its increased prevalence is largely due to societal and environmental factors. Modern lifestyles, modes of transportation, parenting methods, work genres, new media trends and several other factors have favoured an imbalance between required and actual caloric intake. ${ }^{4}$ The major triggers of obesity such as inactivity and unhealthy dietary habits are comparable between Arab Gulf countries and western countries. However, they differ in societal, cultural and environmental triggers such as income, infrastructure, weather, female barriers, living with the extended family, sleep patterns and stress, which are positively correlated with obesity. ${ }^{5-7}$ Among obese children, some recent studies have 
shown the correlation of childhood obesity with age and gender. For instance, obesity is higher among boys than in girls and in older children than in younger children. ${ }^{26}$

Tackling obesity is a complex process that requires multilevel, interdisciplinary-collaborative efforts. Recently, Samara et al identified the main challenges in tackling obesity in the Arab Gulf region. ${ }^{8}$ First, most of the Arab Gulf countries lack important preventive policies such as regular screening and policies for healthy school and work environments. Second, the dynamic interaction between different societal sectors remains inexistent in many Arab Gulf countries. Finally, more effort are needed into translating existing policies and strategies into actionable plans with regular evaluations.

In addition to these challenges, several recent literature reviews have pointed to some gaps in obesity research and evidence from Arab Gulf countries. There has been an increase in obesity-related publications since $2000 .{ }^{9} 10$ However, they lacked good research quality or coverage of all aspects of obesity. ${ }^{11}$ For instance, the main research areas were related to medicine, nutrition, cardiology and biochemistry. ${ }^{10}$ This skewness has disclosed the limited number of studies focused on the social, political, financial and environmental aspects of obesity in Arab Gulf countries. ${ }^{6712}$ Qatar is not an exception in this regard, and despite the high prevalence of childhood and adolescence overweight and obesity, limitations in obesity research exists, highlighting the need for research with better quality, wider representation of population and methodological diversity. ${ }^{13-15}$

\section{Study rationale}

Since 2004, the global political arena has been initiating and promoting adherence to the 'WHO Global Strategy on Diet, Physical Activity and Health', which calls for comprehensive interventions and involvement of all stakeholders. ${ }^{4}$ Of note, Qatar is the first Gulf country to implement the United Nations' approach of 'Health in All Policies' and to develop a well-defined 'National Health strategy 2011-2016', involving the Ministry of Interior, health authorities and the Supreme Council for Family Affairs. ${ }^{8}$ As such, the Supreme Council of Health generated a 20-year blueprint to move from a treatment-based approach to a prevention-based approach, with a focus on promoting healthier diets and increasing physical activity. ${ }^{16}$ Several national guidelines were issued to promote physical activities, healthy diet and healthy school environments, in addition to enhancing the infrastructure of green parks, walkways and cycling tracks. ${ }^{2}$ Obesity prevention is a key goal of the Qatar National Health Strategy (2018-2022), and Qatar is planning to develop a comprehensive strategy for obesity prevention. ${ }^{17}$ For these reasons, adopting a preventive and evidence-based approach requires quality research and relevant evidence. ${ }^{16}$ The research community in Qatar is actively producing significant data through projects such as the biobank ${ }^{17}$ and the Qatar Active Healthy Kids Report Card. ${ }^{14}$ However, the full scope of the available data on Qatar is absent. Most of the existing reviews on obesity provide general information on Arab Gulf countries ${ }^{5611}$ or address Qatar specifically; however, the latter are either nonsystematic or outdated. ${ }^{13}$

This will be the first scoping review to systematically investigate existing literature on obesity in Qatar, with a specific focus on children and adolescents. It will support the efforts of obesity prevention by providing an up-todate research map that highlights the knowledge gaps and methodological range. In addition, this review will be the first scoping review that analyses how obesity research has evolved in Qatar since 2000, while highlighting research gaps and required developments.

\section{Study objectives and questions}

Objectives

1. To examine the nature, diversity and representativeness of the methodologies used in available evidence/ knowledge on obesity among children and adolescents in Qatar

2. To systematically map and chart the available literature on obesity among children and adolescents in Qatar.

3. To identify and analyse research gaps in obesity among children and adolescents in Qatar

4. To track the evolution of research on obesity among children and adolescents in Qatar over time.

\section{Questions}

1. How is research on obesity among children and adolescents in Qatar conducted?

2. What is the available data on obesity among children and adolescents in Qatar?

3. How has research on obesity among children and adolescents in Qatar evolved?

\section{METHODS AND ANALYSIS}

The guidelines of Joanna Briggs Institute (JBI) for knowledge synthesis ${ }^{18}$ and Preferred Reporting Items for Systematic Reviews and Meta-Analyses Extension for Scoping Reviews ${ }^{19}$ will be used to conduct this scoping review. Accordingly, the inclusion criteria, search strategy, source of evidence selection, data extraction, evidence analysis and presentation of the results are described.

\section{Inclusion criteria}

This review will include any literature (in Arabic or English) on the concept of obesity/overweight among the population of children and/or adolescents in the context of Qatar published between January 2000 and 31 December 2021. The start date for choosing literature is based on the fact that obesity research has been predominantly carried out since $2000 .{ }^{910}$ A review of the past 22 years characterised by increased research activity related to obesity is sufficient to fulfil this review's purpose and objectives. 


\section{Exclusion criteria}

- Studies focusing on obesity in general population of Qatar and not specifically on obesity in children or adolescents.

- Study items without specific data on Qatar but rather general/combined results for Gulf Cooperation Council or the Middle East and North Africa (MENA).

- Literature where obesity is a secondary topic and not the main focus; for example, diabetes and cancer research.

- Studies in languages other than English or Arabic

- Theses and dissertations.

\section{Piloting}

As per JBI recommendations, ${ }^{18}$ a random sample of 20 titles/abstracts will be screened by two independent researchers using the inclusion criteria to check for any discrepancies that could lead to a modification of the inclusion criteria. Disagreement will be managed by discussion between the two reviewers and if the disagreement remains, a third reviewer will be approached to resolve the matter and decide on the final inclusion.

\section{Search strategy}

As recommended by JBI, ${ }^{18}$ a three-step search strategy will be used. In addition, a fourth step will be added to identify grey literature. The steps are as follows:

First, an initial limited literature search will be conducted. Then, the keywords from the text and the abstract and the index terms in each article will be analysed.

Second, all identified keywords and index terms will be used as search terms in all included databases (check online supplemental appendix 1 for full search strategy).

Third, reference lists of the identified articles will be checked for more relevant materials that fulfil the inclusion criteria.

Fourth, any grey literature will be identified via three channels (check online supplemental appendix 2 for grey literature search strategy):

1. The reference lists of identified materials.

2. Contacting some corresponding authors of the identified studies for provision of any recent grey literature on the topic. They will be contacted directly or via social media.

3. Searching governmental, non-governmental and relevant official international websites via Google.

\section{Source of evidence selection}

Based on an assessment of more than 20 systematic and scoping reviews on Qatar/MENA obesity/public health issues, the most common and frequently used data sets were PubMed and Embase.

Based on existing literature, research in this area is uncommon and is mostly performed from a medical perspective. ${ }^{6} 71112$ In this scoping review, the aim is to systematically confirm this by conducting a comprehensive systematic search of diverse databases. Therefore,
PubMed, Embase, Web of Science, IBSS, SCOPUS, J-STOR and APA PsycNET will be searched. In addition, the following Arabic databases will also be searched: Dar Almanthomah, Dar Almanha, and E-Marefah.

\section{Search terms}

The search terms have been developed with the advice of a specialist librarian. Searches will be performed for these terms in the title, abstract, and keywords (check online supplemental appendix 1 for full search strategy).

Qatar* and

Obes* OR body mass index OR bmi OR weight OR overweight OR body composition OR fat* OR waist OR circumference OR oversize* adolescent* OR teen* OR student* OR child* OR kid* OR young* OR youth* OR infant* OR toddler* OR newborn eat* OR diet* OR food OR nutrition* OR calorie* active* OR mobile* OR lifestyle* OR sport* OR physical* OR move* OR exercise*

\section{Screening}

The screening stages will be guided by the inclusion and exclusion criteria described above. Two independent reviewers will screen the titles/abstracts and full texts. Disagreement will be managed by discussion between the two reviewers and if the disagreement remains, a third reviewer will be approached to resolve the matter and decide on the final inclusion.

\section{Data extraction}

The System for the Unified Management, Assessment and Review of Information (SUMARI)-JBI software will be used for data extraction. ${ }^{20}$ The key information that will be extracted is:

1. Author(s).

2. Year of publication.

3. Country of origin (where the source was published).

4. Type of publication.

5. Aims/purpose/objectives.

6. Theoretical framework (if applicable).

7. Methodology/methods (if applicable).

8. Population and sample size (if applicable).

9. Any involvement with participants or other stakeholders.

10. Intervention type (if applicable).

11. Duration of the intervention (if applicable).

12. Key findings/outcomes.

\section{Analysis of the evidence}

Data will be analysed descriptively and analytically using simple frequency counts and qualitative content analysis. Critical appraisal will not be performed as this is beyond the scope of scoping reviews.

\section{Presentation of the results}

In addition to a narrative representation of the results, maps, tables and diagrams may be used to present results, depending on the type and amount of data extracted.

\section{Patient and public involvement}

This review will be grounded on patient and public involvement across all the processes: needs assessment, review 
questions, coverage of grey literature, analysis, dissemination and knowledge mobilisation. Prior to designing the protocol, I conducted several interviews with patients, physicians, academics and policymakers: all these stakeholders emphasised the need for this review. From these interviews, the lack of most recent data and policies, and patient and public involvement in designing and implementing obesity research and interventions were the key focus areas. Moreover, most interviewees questioned the diversity of the available data and the comprehensive coverage of obesity issues, research methods, population samples and interventions in recent publications on obesity. During the screening and selection process, I will consult key actors directly to ensure extensive coverage of grey literature. Furthermore, an online call for grey literature nomination will be posted in relevant social media communities, mainly Twitter and LinkedIn, since these are preferred social media platforms among academicians. In the last stage of this review, the outcomes will be discussed in several focus groups consisting of diverse patient groups and their families, physicians, researchers and policymakers. The purpose of these focus group discussions is to seek validation of our findings and unaddressed research gaps that will aid future research. The dissemination activities will be carried out in collaboration with patients for increased impact and relevance. Finally, an accessible online community will be established to work on the research gaps identified in this review.

\section{ETHICS AND DISSEMINATION}

Ethics approval is not required as data will be collected from publicly available published research. The results of this review will be shared with the public through a peerreviewed publication, national and international conferences, workshops and knowledge mobilisation activities among key stakeholders. This review will also contribute to the author's $\mathrm{PhD}$ thesis and will be deposited in the information system of the University of Bath.

\section{Twitter Mariam Alisi @MariamAlisi}

Acknowledgements I want to thank all the patients and the public members who shared their experiences and opinions that led to the formation of this protocol. Also, I would like to recognise the consultative role of my PhD supervisors, Dr Rana Jawad and Professor Rachel Forrester-Jones. In addition, I would like to thank Mr Peter Bradley, the subject librarian for Health and Social \& Policy Sciences at the University of Bath for his invaluable assistance.

Contributors MA developed and planned the scoping review and wrote the protocol manuscript.

Funding The authors have not declared a specific grant for this research from any funding agency in the public, commercial or not-for-profit sectors.

Competing interests None declared.

Patient consent for publication Not applicable.

Provenance and peer review Not commissioned; externally peer reviewed.

Supplemental material This content has been supplied by the author(s). It has not been vetted by BMJ Publishing Group Limited (BMJ) and may not have been peer-reviewed. Any opinions or recommendations discussed are solely those of the author(s) and are not endorsed by BMJ. BMJ disclaims all liability and responsibility arising from any reliance placed on the content. Where the content includes any translated material, BMJ does not warrant the accuracy and reliability of the translations (including but not limited to local regulations, clinical guidelines, terminology, drug names and drug dosages), and is not responsible for any error and/or omissions arising from translation and adaptation or otherwise.

Open access This is an open access article distributed in accordance with the Creative Commons Attribution Non Commercial (CC BY-NC 4.0) license, which permits others to distribute, remix, adapt, build upon this work non-commercially, and license their derivative works on different terms, provided the original work is properly cited, appropriate credit is given, any changes made indicated, and the use is non-commercial. See: http://creativecommons.org/licenses/by-nc/4.0/.

ORCID iD

Mariam Alisi http://orcid.org/0000-0002-8623-4347

\section{REFERENCES}

1 World Population Review. Most obese countries population, 2019. Available: http://worldpopulationreview.com/countries/most-obesecountries [Accessed 8 Sep 2021].

2 Al-Thani M, Al-Thani A, Alyafei S, et al. The prevalence and characteristics of overweight and obesity among students in Qatar. Public Health 2018;160:143-9.

3 Farrag NS, Cheskin LJ, Farag MK. A systematic review of childhood obesity in the middle East and North Africa (Mena) region: prevalence and risk factors meta-analysis. Adv Pediatr Res 2017:4:8.

4 World Health Organization (WHO). Obesity and overweight, 2018. Available: https://www.who.int/en/news-room/fact-sheets/detail/ obesity-and-overweight [Accessed 8 Sep 2021].

5 Balhareth A, Meertens R, Kremers S, et al. Overweight and obesity among adults in the Gulf states: a systematic literature review of correlates of weight, weight-related behaviours, and interventions. Obes Rev 2019;20:763-93.

6 Al Yazeedi B, Berry DC. Childhood overweight and obesity is increasing in Gulf cooperation Council countries: a review of the literature. J Transcult Nurs 2019;30:603-15.

7 AlAbdulKader AM, Tuwairqi K, Rao G. Obesity and cardiovascular risk in the Arab Gulf states. Curr Cardiovasc Risk Rep 2020;14:1-9.

8 Samara A, Andersen PT, Aro AR. Health promotion and obesity in the Arab Gulf states: challenges and good practices. $J$ Obes 2019;2019:1-6.

9 Sweileh WM, Zyoud Sa'ed H, Al-Jabi SW, et al. Quantity and quality of obesity-related research in Arab countries: assessment and comparative analysis. Health Res Policy Syst 2014;12:1-10.

10 AlMarri F, Al Sabah S, Al Haddad E, et al. A call for more research from the Arabian Gulf. Obes Surg 2017;27:2034-43.

11 Al Hammadi H, Reilly J. Prevalence of obesity among school-age children and adolescents in the Gulf cooperation Council (GCC) states: a systematic review. BMC Obes 2019;6:3.

12 Hoque R, Strotheide E, Saquib J, et al. Assessment of nationally representative dietary studies in the Gulf cooperation Council: a scoping review. PeerJ 2020;8:e10163.

13 Mandeya J, Kridli SA-O. Childhood overweight and obesity in Qatar: a literature review. Avicenna 2014;2014:2.

14 Al-Kuwari MG, Ibrahim IA, Hammadi EMA, et al. Results from Qatar's 2016 active healthy kids report card on physical activity for children and youth. JPhys Act Health 2016;13:S246-50.

15 Khondaker MTI, Khan JY, Refaee MA, et al. Obesity in Qatar: a case-control study on the identification of associated risk factors. Diagnostics 2020;10:883.

16 Chouchane L, Mamtani R, Al-Thani MH, et al. Medical education and research environment in Qatar: a new epoch for translational research in the middle East. $J$ Trans/ Med 2011;9:16.

17 Taheri S, Al-Thani M. Obesity in Qatar: current and future strategies. Lancet Diabetes Endocrinol 2021;9:561-2.

18 Peters MDJ, Godfrey C, McInerney P. Chapter 11: Scoping Reviews. In: Aromataris E, Munn Z, eds. JBI manual for evidence synthesis, $J B I, 2020$. https://synthesismanual.jbi.global

19 Tricco AC, Lillie E, Zarin W, et al. PRISMA extension for scoping reviews (PRISMA-ScR): checklist and explanation. Ann Intern Med 2018;169:467-73.

20 Munn Z, Aromataris E, Tufanaru C, et al. The development of software to support multiple systematic review types: the Joanna Briggs Institute system for the unified management, assessment and review of information (JBI SUMARI). Int J Evid Based Healthc 2019;17:36-43. 